Journal of the Electrochemical Society, Vol. 142, No. 8, 1995, pp. 2591-2594.

ISSN: (Print 0013-4651) (Online 1945-7111)

DOI: $10.1149 / 1.2050058$

http://www.electrochem.org/

http://scitation.aip.org/JES

http://scitation.aip.org/getpdf/servlet/GetPDFServlet?filetype=pdf\&id=JESOAN000142000008002591000001\&idty pe $=$ cvips \&prog $=$ normal

(C) The Electrochemical Society, Inc. 1995. All rights reserved. Except as provided under U.S. copyright law, this work may not be reproduced, resold, distributed, or modified without the express permission of The Electrochemical Society (ECS). The archival version of this work was published in Journal of the Electrochemical Society, Vol. 142, No. 8, 1995, pp. 2591-2594.

\title{
Corrosion and Protection of a Conductive Silver Paste
}

\author{
V. Brusic, G. S. Frankel, J. Roldan, and R. Saraf \\ IBM Research Division, T.J. Watson Research Center, Yorktown Heights, New York
}

\begin{abstract}
One of the possible uses for a conductive paste is as an adhesive in interconnect technology that could replace $\mathrm{PbSn}$ solder. The interconnections are expected to perform under a variety of environmental conditions, and with an applied voltage. Thus knowledge of their corrosion and dissolution resistance is of utmost importance. This is a study of the dissolution and protection of polymer/metal composite films, prepared with a high loading of silver or gold particles. Electrochemical tests were conducted in a droplet of triple-distilled water with or without benzotriazole (BTA) and BTA derivatives. Results indicate that, in spite of some protection obtained by the polymer, silver paste dissolution at high anodic potentials is rapid, reaching values of $10^{-1} \mathrm{~A} / \mathrm{cm}^{2}$, which corresponds to a catastrophic silver removal rate of at least $35.6 \mathrm{~nm} / \mathrm{s}$. With a reservoir of azole in the corrosive environment, this rate can be reduced by up to five orders of magnitude. This azole effect greatly reduces the probability of electrolytic silver migration, but the Ag dissolution rate is still higher than the anodic activity shown by Au paste under the same conditions.
\end{abstract}

\section{Introduction}

Insulating thermoset and thermoplastic polymers can be made conductive by loading them with metallic powder particles such as silver, copper, or gold. The resulting conductive paste has many potential applications. Such a material can be used for electrostatic discharge protection, electromagnetic interference shielding, and as adhesive for interconnect technology that could replace $\mathrm{PbSn}$ solder. The widespread use of $\mathrm{Pb}$ containing solder in electronics is an environmental concern, and regulations banning $\mathrm{Pb}$ are being considered. Whereas paste conductivity is the critical property in interconnection applications, the corrosion and dissolution resistance are also of the utmost importance. In order to be conductive, the paste has to have a high loading level, i.e., it should contain more than 50 weight percent (w/o) of the metal powder. Interconnections are expected to perform under a variety of environmental conditions, and with an applied voltage, where metal dissolution can result in electrolytic migration, dendrite formation, and eventually electrical shorts or opens. Thus, the susceptibility of the paste to dissolve under an applied potential indicates a potential problem for a device in operation.

This is a study of the dissolution and protection of polymer/metal composite (PMC) films, prepared in our laboratory with a high loading of silver particles. Electrochemical tests were conducted in a droplet of triple-distilled water with or without benzotriazole (BTA) and BTA derivatives. Same of the patent literature, ${ }^{1}$ as well as experience in our laboratory, has indicated that $\mathrm{Ag}$ forms a thin layer of $\mathrm{Ag}$-BTA in a manner similar to $\mathrm{Cu}$ under the same 
conditions. Yet, the protective nature of Ag-BTA films is not well documented. Results are discussed in terms of observed protection offered by BTA and compared to the results obtained on a similar paste formulated with Au particles.

\section{Experimental}

The PMC samples were prepared on a glass substrate as blanket films with dimensions of $1.9 \times 3.2 \mathrm{~cm}$ and a thickness range from 25 to $40 \mu \mathrm{m}$. Ag or Au particles (with a typical loading factor of 88 w/o) were used in the preparation of the paste. Some of the variations used in the PMC preparation include (i) additions of 1000 ppm BTA to the paste formulating mixture, (ii) 10 min immersion into an aqueous solution of $10^{-2} \mathrm{M}$ BTA, and (iii) $3 \mathrm{~min}$ dip into an alcohol solution of BTA or $5 \mathrm{CH}_{3} \mathrm{BTA}$ in concentrations of 0.02 and $0.2 \mathrm{M}$ prior to electrochemical evaluation of the corrosion behavior in a selected electrolyte. The temperature of the alcoholazole solution was controlled in the range of room temperature to $60^{\circ} \mathrm{C}$.

Plating tape was used to expose an area of $0.32 \mathrm{~cm}^{2}$ for electrochemical testing. The electrochemical cell also contained a $\mathrm{Pt}$ mesh as a counterelectrode and a mercurous sulfate electrode (MSE, with saturated solution of $\mathrm{K}_{2} \mathrm{SO}_{4}$ ) as a reference electrode, that were separated by a filter paper. This cell design, described elsewhere, ${ }^{2}$ was proven suitable for use with a small volume of an electrolyte, $0.01 \mathrm{ml}$ or so, and with electrolytes that are normally difficult to handle or have low conductivity. Because of the closeness of the electrodes, the cell is suitable for tests in water without significant ohmic potential drop. The use of pure water as an electrolyte mimics the conditions that apply in processing (such as product rinsing) or in exposure to a humid atmosphere. The electrochemical tests consisted of corrosion potential measurement, which was interrupted in regular time intervals by measurement of linear polarization to determine the corrosion rate. After about $10 \mathrm{~min}$, when the open potential reached a stable value (changing no more than $2 \mathrm{mV} / \mathrm{min}$ ), a potentiodynamic sweep was applied. A sweep rate of $1 \mathrm{mV} / \mathrm{s}$ was used, starting $250 \mathrm{mV}$ below the corrosion potential and reaching $1.3 \mathrm{~V}$ vs. MSE. In several tests, dissolution of Ag paste was examined by an application of a constant anodic potential and a continuous recording of the current, i.e., with potentiostatic control. The electrolyte was water, with or without BTA or an azole derivative.

Most of the electrochemical tests were conducted within hours of the paste preparation. Some of the experiments were performed after months of sample storage in either open containers, or in closed containers containing a dry $5 \mathrm{CH}_{3}$-BTA reservoir.

In a few experiments, using Ag foil, ellipsometry was used to determine the presence and thickness of Ag-azole films on the silver surface, and time-of-flight static secondary ion mass spectroscopy (SIMS) was employed to determine the film composition.

\section{Results and Discussion}

Anodic dissolution of silver.-Although noble, silver dissolves in water saturated with air at a corrosion rate of about $1 \times 10^{-7} \mathrm{~A} / \mathrm{cm}^{2}$ or $0.002 \mathrm{~nm} / \mathrm{min}$. The results obtained with a pure silver foil are shown in Fig. 1. With increased anodic potential, silver dissolution rapidly increases, in spite of the thermodynamic prediction of higher valent silver oxide formation. ${ }^{3}$ Apparently none of the oxides that are expected to form, $\mathrm{Ag}_{\mathrm{a}} \mathrm{O}, \mathrm{Ag}_{2} \mathrm{O}_{2}$, and $\mathrm{Ag}_{2} \mathrm{O}_{3}$, are truly protective, and at high anodic potentials Ag dissolves with a current that (for the given experimental area) exceeds the measurable limit of the EG\&G Princeton Applied Research 273 potentiostat. The highest measured current density of $10 \mathrm{~A} / \mathrm{cm}^{2}$ corresponds to the dissolution rate of $3.6 \times 10^{+3} \mathrm{~nm} / \mathrm{s}$ (with an assumption that at these anodic potentials Ag dissolves with a three-electron exchange). 
These very high anodic rates measured in water confirm what is already known from the anodic behavior of silver in aqueous solution ${ }^{4}$ and vast experience in electronic industry ${ }^{5}$ : in the presence of humidity and bias, silver forms poorly passive, fairly soluble silver oxides. Dissolved ions increase the conductivity of the adsorbed water layers, easily migrate under the influence of the electric field, and provide reactants for the cathodic reaction, which is deposition of $\mathrm{Ag}$ dendrites. Thus the magnitude of the anodic current in the water droplet is a relative measure of the vulnerability to form electrical shorts in interconnection applications.

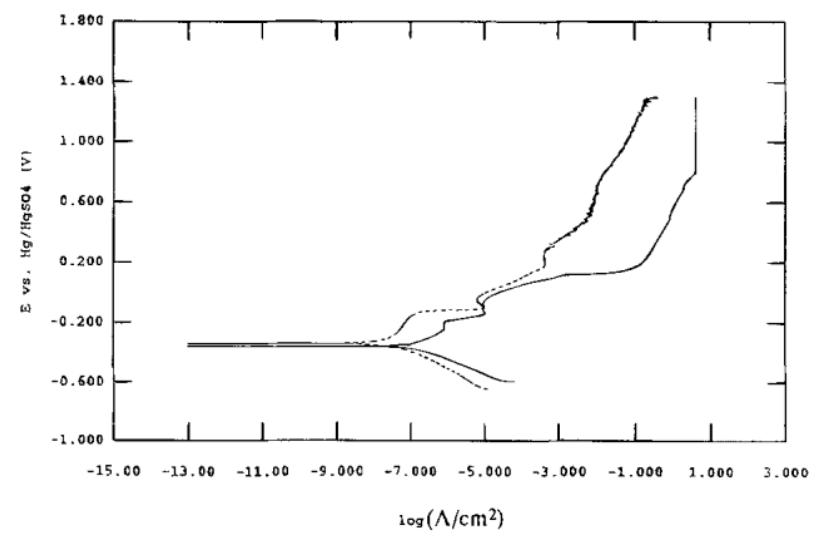

Fig. 1. Potentiodynamic polarization curves for an $\mathrm{Ag}$ foil (solid line) and $\mathrm{Ag}$ paste (dashed line) samples measured in a droplet of water.

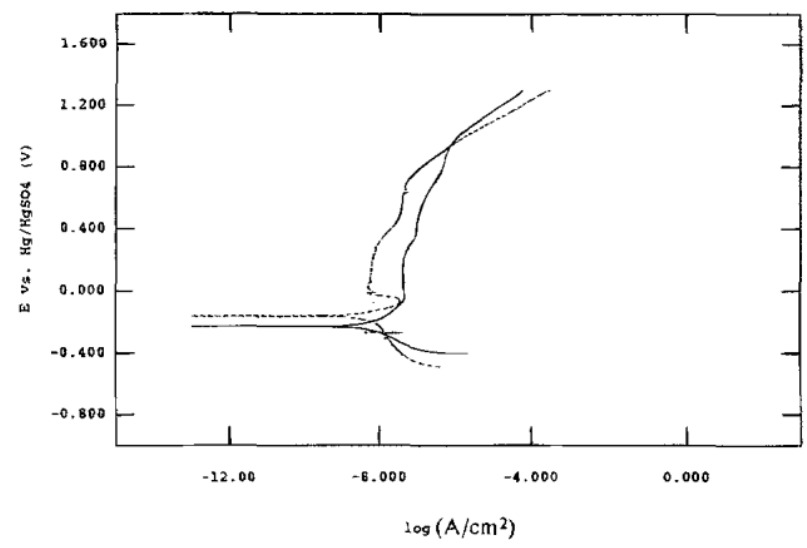

Fig. 2. Potentiodynamic polarization curves measured on Au film (solid line) and Au paste (dashed line).

Metal coverage in the conductive paste.-The corrosion rate measured as current density (per geometric area) on Ag paste is about an order of magnitude smaller than that of Ag foil, Fig. 1. This indicates that the actual area of the metal available for reaction is about $10 \%$ of the available area, or that the polymeric coverage for this particular paste is about $90 \%$.

Dissolution of Au paste. - In comparison to the Ag paste, the PMC film with Au particles has significantly lower currents both at the corrosion potential as well as at high anodic potentials, Fig. 2. As in the case of Ag, the apparent metal coverage in the paste is about $90 \%$, judging from the dissolution rates at the corrosion potential. The Au paste is possibly contaminated with some $\mathrm{Ag}$ (from previous milling of the $\mathrm{Ag}$ paste during formulation) that could explain the observed current peak below $0 \mathrm{~V}$. Both $\mathrm{Au}$ and Au paste show relatively low 
anodic currents below about $0.8 \mathrm{~V}$. The measured current density of about $10^{-7} \mathrm{~A} / \mathrm{cm}^{2}$ is about an order of magnitude higher than the current due to charging of the double layer during the scan. For a double layer capacitance of $20 \mathrm{n}, \mathrm{F} / \mathrm{cm}^{2}$, the capacitive current would be approximately $2 \times$ $10^{-8} \mathrm{~A} / \mathrm{cm}^{2}$. If the anodic current were caused by Au dissolution, an average Au removal rate would be $1.1 \times 10^{-3} \mathrm{~nm} / \mathrm{s}$. The increase of the anodic current at potentials above $0.8 \mathrm{~V}$ is attributed to the evolution of oxygen, which proceeds much slower than does the dissolution of $\mathrm{Ag}$ at the same potentials.

Effect of BTA and $5 \mathrm{CH}_{3}$ BTA on dissolution of Ag.-Potentiodynamic curves measured in water with $10^{-2} M$ BTA show remarkable reduction of the Ag dissolution rate, Fig. 3. An increase of the corrosion potential and a decrease of the corrosion rate suggest that the presence of this inhibitor slows down preferentially the anodic reaction in the overall corrosion process, similar to the reports for the effect of BTA on $\mathrm{Cu}$ corrosion. ${ }^{6}$ In contrast to the case of copper, however, BTA is even more effective at high anodic potentials, with the average dissolution rate being more than four orders of magnitude lower than that measured in water alone. Moreover, the anodic dissolution rate of $\mathrm{Ag}$ paste in the presence of BTA is almost as slow as the rate measured on pure Au paste.

Ellipsometric data on $\mathrm{Ag}$ foil that was immersed in the aqueous BTA solution, rinsed in water, and $\mathrm{N}_{2}$ dried, show a formation of a surface film with a thickness of about $3 \mathrm{~nm}$. This is very close to the thickness of $\mathrm{Cu}$-BTA film that is normally formed on oxidized $\mathrm{Cu}$ surface under similar circumstance, i.e., $2.5 \mathrm{~nm},{ }^{6,7}$ and thicker than the measured BTA films that weakly adsorb on metals, such as Fe, Ni, or Al, where the BTA film thickness is less than $0.5 \mathrm{~nm}$. Time-offlight static SIMS data indicate that the film is $\mathrm{Ag}$-BTA. $\mathrm{Ag}^{+}$ions most likely occupy the same site in the benzotriazole ring as the $\mathrm{Cu}^{+}$ions in $\mathrm{Cu}$-BTA complex. However, in contrast to $\mathrm{Cu}-$ BTA on $\mathrm{Cu}$, thin Ag-BTA on Ag shows only a marginal protection against corrosion when retested for the corrosion in water without a BTA reservoir. At higher anodic potential, effects of the azoles were observed only when these inhibitors were present in a solution as a reservoir.

As the thermal instability of BTA and its derivatives is well documented, ${ }^{6,7}$ additions of BTA to the paste during fabrication were not expected to be successful in a preparation of a built-in azole reservoir. This was indeed confirmed by experiments. However, immersion of paste into an alcohol-azole solution resulted in a surface film that offered a significant protection in subsequent exposure to water, Fig. 3. Visual observation and ellipsometric investigation of the Ag foil immersed in an alcohol-azole solution and dried (without rinsing) show a presence of a thick, white film. After rinsing in alcohol, the foil retains its shine, and ellipsometry indicates the presence of a film that is about $3 \mathrm{~nm}$ thick. Thus, as well as forming a thin Ag-BTA complex, the process apparently blocks the pores in the paste by a precipitated, noncomplexed, azole.

The beneficial effect of the built-in azole reservoir increased with an increase in concentration and temperature of the azole-alcohol solutions. The most effective, repro-ducibly measured protection, was observed with concentrated azole solutions $(0.2 \mathrm{M}$ or more $)$ applied at 50 or $60^{\circ} \mathrm{C}$ for $3 \mathrm{~min}$. Of the two azoles that were tested, $5 \mathrm{CH}_{3} \mathrm{BTA}$ offered a more effective protection. In the best cases, such reservoirs decreased the anodic dissolution in water four (with BTA) to five (for $5 \mathrm{CH}_{3} \mathrm{BTA}$ ) orders of magnitude. Under the best conditions, Ag dissolution approached the values measured on $\mathrm{Au}$ paste in water, with the $\mathrm{Au}$ paste still being better by an order of magnitude. 


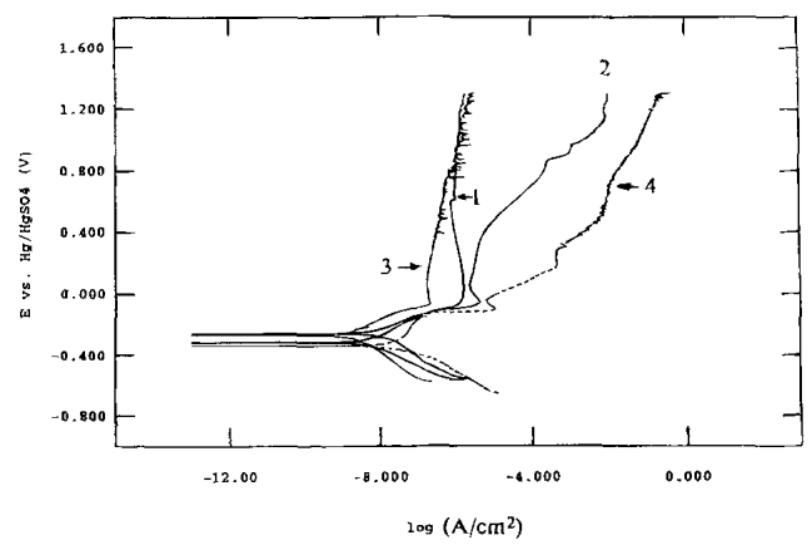

Fig. 3. Potentiodynamic polarization curves measured on Ag paste in aqueous solution of $0.01 M$ BTA (1), and in triple distilled water (2 and 3) on Ag paste immersed for 3 min into an alcohol azole solution kept at $60^{\circ} \mathrm{C}$ prior to the measurements in water (2, alcohol contained $0.2 M$ BTA; 3 , alcohol contained $0.2 M 5 \mathrm{CH}_{3} \mathrm{BTA}$ alcohol). Data obtained on untreated Ag paste in water are plotted for a comparison (4).

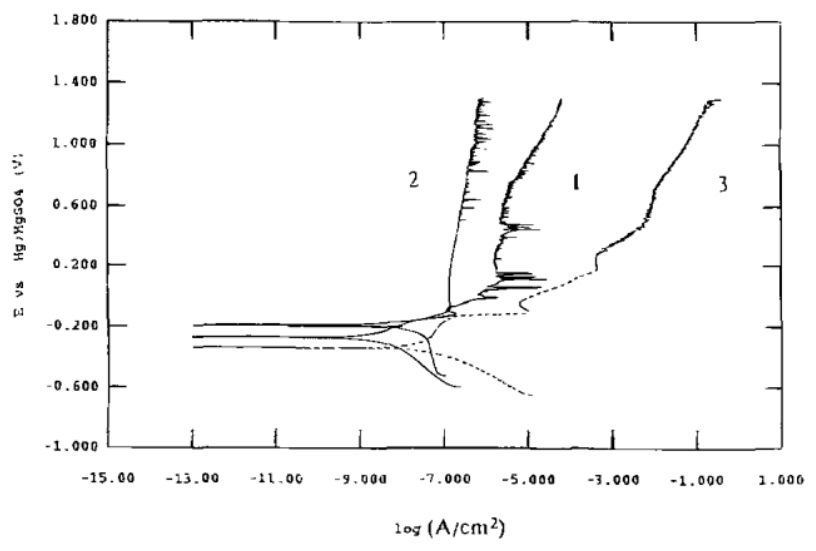

Fig. 4. Potentiodynamic curves measured on $\mathrm{Ag}$ paste treated with $5 \mathrm{CH}_{3}$-BTA/alcohol mixture measured after 4 months of storage in an open container (curve 1). Results on unprotected paste (curve 3) and on protected paste, measured hours after the treatment (curve 2) are given for a comparison.

One of the most important properties of the paste, its electrical conductivity, is not changed by application of the azole reservoir. There are, however, some possible shortcomings of this treatment, such as its effects on the appearance and on the surface conductivity of the treated part. The altered surface appearance is a consequence of the azole application without rinsing, with a residual, nonuniform, film present over the entire exposed area. Its effect on surface conductivity cannot be easily predicted. By itself, this film is not conducting, and it should not cause electrical failures in the circuits. Dissolved in water, BTA increases solution conductivity: $0.01 M$ BTA is 10 times more conductive than water alone. Thus, when the parts get exposed to an elevated relative humidity, dissolution of the azole in the adsorbed water layer should increase the conductivity of that film. The surface wetting, however, is expected to decrease for the azole-treated surface. ${ }^{8}$ The overall effect of the azole film on the behavior of an electronic circuit exposed to humidity and bias will be experimentally evaluated at a future time. The potentiodynamic curves recorded on samples that were treated with the azole-alcohol solutions show some level of current instability (Fig. 3-5). The origin of the recorded noise is not 
fully understood, but it might be rooted in somewhat dynamic wetting of the rough paste surface, a fast localized dissolution of $\mathrm{Ag}$, and a delayed reaction of $\mathrm{Ag}$ ions with dissolved azole. In contrast, anodic currents obtained on azole-alcohol treated samples in potentiostatic experiments are very stable. Figure 6 shows the current density-time curves for a sample treated with $5 \mathrm{CH}_{3}$ BTA and stored in a closed container with a surplus of azole for more than 6 months and then oxidized in water at 0.5 and $1.0 \mathrm{~V} \mathrm{MSE}$, respectively, for $20 \mathrm{~min}$. Similar data for untreated paste oxidized at $0.15 \mathrm{~V}$ are shown in Fig. 7. The steady-state current densities follow closely the behavior predicted from the potentiodynamic polarization curves, Fig. 1 and 5. Oxidation of Ag is fast, causing noticeable surface roughening, dissolution and precipitation of silver oxides, all resulting in a large variation in current density. Dissolution rate is of the order of magnitude of $0.15 \mathrm{~A} / \mathrm{cm}^{2}$ at $0.15 \mathrm{~V}$, Fig. 7. (In comparison, currents obtained on Ag foil are higher and even more noisy.) The azole-alcohol treated sample shows a dissolution rate below $1 \times 10^{-3} \mathrm{~A} / \mathrm{cm}^{2}$ at $0.15 \mathrm{~V}, 3.7 \times 10^{-8} \mathrm{~A} / \mathrm{cm}^{2}$ at $0.5 \mathrm{~V}$, and $3.1 \times 10^{-7} \mathrm{~A} / \mathrm{cm}^{2}$ at $1.0 \mathrm{~V}$. Thus, again, the reduction of the dissolution rate provided by the azole-alcohol treatment is at least five orders of magnitude.

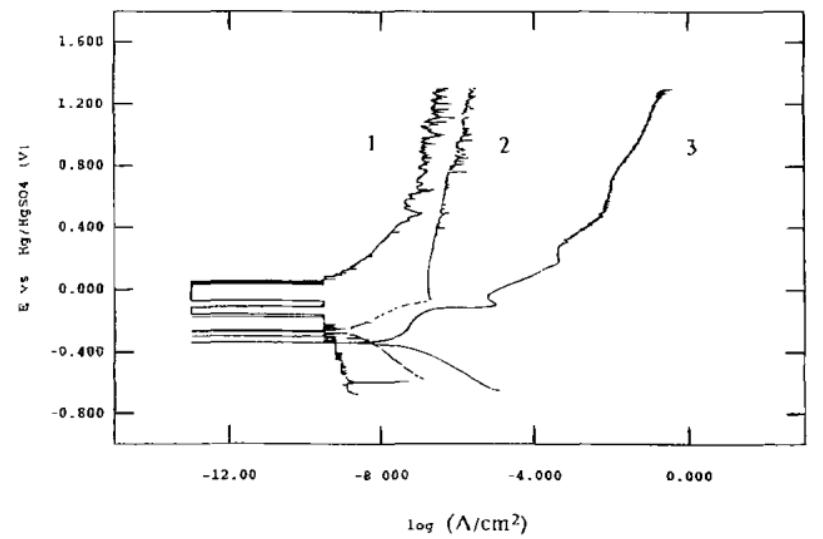

Fig. 5. Potentiodynamic curves measured on $\mathrm{Ag}$ paste treated with $\mathrm{CH}_{3}-\mathrm{BTA}$ /alcohol mixture measured after 4 months of storage in a closed container with a surplus of $\mathrm{CH}_{3}$-BTA powder (curve 1). Results on unprotected paste (curve 3) and on protected paste, measured hours after the treatment (curve 2) are given for a comparison.

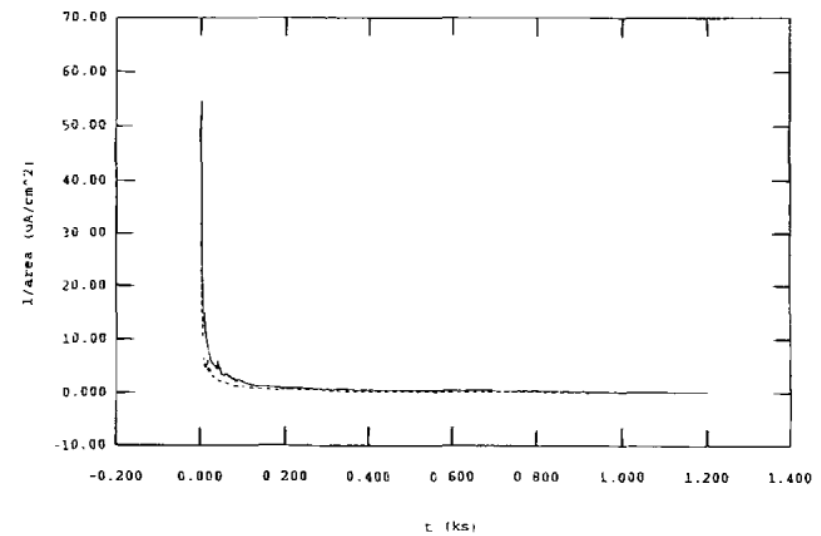

Fig. 6. Variation of current density with time during potentiostatic oxidation of azole-alcohol treated paste at $0.5 \mathrm{~V}$ (dashed line) and 1.0 V MSE (solid line) in water. Sample was stored in an azole containing container for 6 months prior to the electrochemical test. 
Both BTA and $5 \mathrm{CH}_{3}-\mathrm{BTA}$ have relatively high vapor pressure $\left(0.09\right.$ and $0.02 \mathrm{~mm} \mathrm{Hg}$ at $40^{\circ} \mathrm{C}$, respectively), and the paste protection by noncomplexed azoles should decrease with time. Thus the electrochemical tests were repeated four months after the application of the $5 \mathrm{CH}_{3}-\mathrm{BTA} /$ alcohol treatment with some films being stored in an open container and some in a closed container that also had an added $\mathrm{CH}_{3}$-BTA powder. The results are given in Fig. 4 and 5. Sample stored in an open container has somewhat higher corrosion rate as well as a dissolution rate at higher anodic potentials than measured immediately after $\mathrm{CH}_{3}$-BTA application, but the dissolution is still about four orders of magnitude slower than that measured on unprotected paste, Fig. 4. A sample stored in a closed container with a reservoir of $\mathrm{CH}_{3}$-BTA powder has improved its corrosion and dissolution resistance, and its overall activity is smaller than measured on Au paste, Fig. 5.

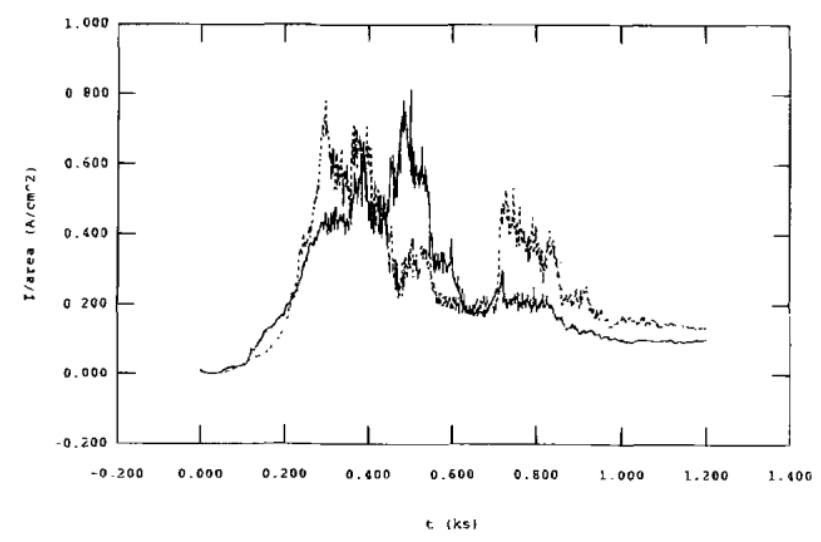

Fig. 7. Variation of current density with time during potentiostatic oxidation of untreated Ag paste at $0.15 \mathrm{~V}$. Results or two separate experiments are plotted.

\section{Conclusions}

1. The polymer used in the paste fabrication provides coverage of more than $90 \%$ for $\mathrm{Ag}$ particles, and a corresponding decrease of the $\mathrm{Ag}$ corrosion and dissolution. In spite of the protection, the dissolution of silver at anodic potentials reaches the current density of $10^{-1} \mathrm{~A} / \mathrm{cm}^{2}$, which far exceeds the rate of oxygen evolution and corresponds to the rate of $\mathrm{Ag}$ removal of at least $35.6 \mathrm{~nm} / \mathrm{s}$.

2. In an aqueous solution containing BTA, the anodic dissolution of Ag particles is greatly reduced. The behavior of the paste becomes similar to that of Au paste except for the fact that even the oxygen evolution reaction is greatly suppressed.

3. A reservoir with either BTA or $5 \mathrm{CH}_{3} \mathrm{BTA}$ can be built into the paste by a short immersion of the paste into an alcohol-azole mixture at elevated temperature followed by nitrogen drying. The bulk of the residual film is composed of noncomplexed BTA or $5 \mathrm{CH}_{3} \mathrm{BTA}$. Because of their relatively high vapor pressure, $(0.09 \mathrm{~mm} \mathrm{Hg}$ for BTA and $0.02 \mathrm{~mm} \mathrm{Hg}$ for $5 \mathrm{CH}_{3} \mathrm{BTA}$ ), these films should disappear with time. $5 \mathrm{CH}_{3} \mathrm{BTA}$ is both a better protector and more stable than BTA. The effective protection of either of these films can be prolonged indefinitely, if they are kept in an enclosure with a solid azole reservoir.

Manuscript submitted Jan. 30, 1995; revised manuscript received April 7,1995. This was Paper 223 presented at the Miami Beach, FL, Meeting of the Society, Oct. 9-14, 1994. 
IBM Research Division assisted in meeting the publication costs of this article.

\section{REFERENCES}

1. S. Kobayashi, M. Itoh, and A. Minato, U. S. Pat. 4,821,148 (1989).

2. V. Brusic, M. Russak, R. Schad, G. Frankel, A. Selius, D. DiMilia, and D. Edmonson, This Journal, 136, 42 (1989).

3. M. Pourbaix, Atlas of Electrochemical Equilibria, Pergamon Press, New York (1966).

4. U. Ebersbach, K. Schwabe, and K. Ritter, Electrochim. Acta, 12, 927 (1967).

5. J. J. Steppan, J. A. Roth, L. C. Hall, D. A. Jeannotte, and S. P. Carbone, This Journal, 134, 175 (1987).

6. V. Brusic, M. A. Frisch, B. N. Eldridge, F P. Novak, F. B. Kaufman, B. M. Rush, and G. S. Frankel, ibid., 138, 2253 (1991).

7. V. Brusic, M. A. Frisch, B. N. Eldridge, F. B. Kaufman, T. A. Petersen, A. Schrott, and G. S. Frankel, in Proceedings of International Symposium on Control of Copper and Copper Alloys Oxidation, Edition de la Revue de metallurgy, Paris (1993).

8. R. R. Thomas, V. A. Brusic, and B. M. Rush, This Journal, 139, 678 (1992). 